\title{
Continuidades y rupturas: La política de salud sexual y reproductiva chilena en cuatro gobiernos
}

\author{
Javiera Cubillos Almendra \\ Universidad Católica del Maule. Talca, Chile. \\ Email: jcubillos@ucm.cl
}

\begin{abstract}
Resumen: Chile fue el primer país latinoamericano en abordar la sexualidad como asunto público (1967), a partir de la instalación de programas internacionales de planificación familiar. Desde entonces, la política pública sobre salud sexual y reproductiva ha seguido una trayectoria fluctuante pudiendo identificarse diferentes énfasis y marcos de interpretación: vinculados al control demográfico, la religión católica, a la salud pública y, más recientemente, a los derechos humanos. Si bien el itinerario de esta política es más extenso, el presente artículo propone un análisis comparativo-a partir de la revisión de documentos políticos y entrevista a actores clave - sobre las iniciativas emprendidas por los últimos cuatro gobiernos (2000-2018), integrando una perspectiva de género. El propósito es identificar énfasis, continuidades y rupturas, en cada mandato presidencial, en la garantía de los derechos sexuales y reproductivos. política.

Palabras clave: Políticas públicas; sexualidad; perspectiva de género; derechos sexuales y reproductivos; ciencia
\end{abstract}

\section{Continuities and breaks: The Chilean sexual and reproductive health policy in four governments}

\begin{abstract}
Chile was the first Latin American country to address sexuality as a public issue (1967), following the installation of international family planning programs. Since then, public policy on sexual and reproductive health has followed a fluctuating trajectory, and different emphases and frameworks of interpretation can be identified: linked to demographic control, Catholic religion, public health and, more recently, human rights. Although the itinerary of this policy is more extensive, this article proposes a comparative analysis -from the review of political documents and interviews with key actors- on the initiatives undertaken by the last four governments (2000-2018), integrating a gender perspective. The purpose is to identify emphasis, continuities and ruptures, in each presidential term, in the guarantee of sexual and reproductive rights.
\end{abstract}

Keywords: Public policy; sexuality; gender approach; sexual and reproductive rights; politic science.

\section{Continuidades e rupturas: A política de saúde sexual e reprodutiva chilena em quatro governos}

Resumo: O Chile foi o primeiro país latino-americano a abordar a sexualidade como uma questão pública em 1967, após a instalação de programas internacionais de planejamento familiar. Desde então, a política pública sobre saúde sexual e reprodutiva seguiu uma trajetória flutuante, e podem ser identificados diferentes ênfases e quadros de interpretação: ligados ao controle demográfico, à religião católica, à saúde pública e, mais recentemente, aos direitos humanos. Embora o itinerário desta política seja mais extenso, este artigo propõe uma análise comparativa -da revisão de documentos políticos e entrevistas com atores chave- sobre as iniciativas empreendidas pelos últimos quatro governos (2000-2018), integrando uma abordagem de gênero. O objetivo é identificar a ênfase, continuidades e rupturas, em cada mandato presidencial, na garantia de direitos sexuais e reprodutivos. 
Palavras-chave: Política pública; sexualidade; perspectiva de género; direitos sexuais e reprodutivos; ciência política.

$$
* * *
$$

\section{Introducción}

El presente artículo analiza la trayectoria de la política de salud sexual y reproductiva en Chile ${ }^{1}$, a partir de los cuatro gobiernos presentes en el periodo 2000-2018, que comprende los periodos presidenciales de Ricardo Lagos Escobar (2000-2006), Michelle Bachelet Jeria (2006-2010), Sebastián Piñera Echeñique (20102014), y el segundo mandato de Michelle Bachelet (2014-2018). La hipótesis que motiva el análisis plantea que no ha existido voluntad política suficiente por parte de los gobiernos para abordar integral y coherentemente el ámbito de la sexualidad, en concordancia con los Derechos Sexuales y Reproductivos (DDSSRR) y una perspectiva de género ${ }^{2}$.

No obstante, se identifican diferentes énfasis, acciones y también similitudes en los cuatro mandatos, que permiten acercarse en mayor o menor medida a las orientaciones proporcionadas por organismos internacionales y a las demandas del movimiento feminista, de mujeres y de la diversidad sexual, quienes han sido los principales precursores de modificaciones a las acciones y las normativas gubernamentales. En este contexto, el análisis busca identificar los principales obstaculizadores y facilitadores para este cometido, los que debiesen tenerse en cuenta al momento de retomarse el debate sobre la garantía de los DDSSRR y el rol que le compete al Estado en esto.

Los antecedentes de la política analizada se remontan a los años 30, cuando el Movimiento Pro Emancipación de la Mujer Chilena (MEMCH) manifiesta la urgencia de contar con métodos anticonceptivos y una regulación que permita evitar abortos clandestinos y sus graves consecuencias (Pieper-Mooney, 2008). Como respuesta, el Estado modificó el Código Sanitario (1931) que incluyó la interrupción del embarazo por razones terapéuticas. Luego, en 1952, cuando se unifica el sistema sanitario a través del Sistema Nacional de Salud, la salud materno-infantil se posiciona como prioridad dada las altas tasas de mortalidad materna. Es en elgobierno de Eduardo Frei Montalva (1964-1970) cuando se crea la primera política de planificación familiar (1967), al amparo del Ministerio de Salud (MINSAL), y el primer programa de educación sexual -Programa de vida familiar y educación sexual—, que estuvo a cargo del Ministerio de Educación (MINEDUC) (Olavarría, 2005). Tales intervenciones gubernamentales -aunque fueron promovidas por organismos internacionales desde interpretaciones higienistas y neomalthusianas sobre desarrollo - inauguraron una nueva fase en el campo político de la sexualidad y, a pesar de provocar reacciones contrarias desde sectores conservadores, en general, fueron iniciativas bien recibida (Bonan, 2004; Labarca, 2008; Zárate y González, 2015).

Durante el breve gobierno de Salvador Allende (1971-1973) se mantuvo la línea definida por la política de Frei Montalva, con énfasis en la salud pública. Dichas acciones, no obstante, fueron interrumpidas por la dictadura cívico-militar de Augusto Pinochet (1973-1990), donde además de desmontarse los programas de planificación familiar, reformarse el sistema sanitario (1979) y promoverse una política pro-natalista, se penaliza el aborto en toda circunstancia (1989).

En la transición a la democracia, a partir de las presiones desde la sociedad civil y organismos internacionales, el Estado ha manifestado interés por avanzar en la garantía de derechos a toda la ciudadanía, incluido los DDSSRR, y cumplir con los acuerdos establecidos por el Derecho Internacional. Bajo tales lineamientos, Chile ha experimentado ciertas mejoras. Sin embargo, el proceso hacia la definición de una política al respecto no ha estado exento de obstáculos y polémicas. Los principales frenos para avanzar en nuevas garantías en el ámbito de la sexualidad responden a una historia marcada por la dictadura cívico-militar (19731989), periodo en el que el Estado asumió un rol subsidiario, se promovió una política pro-natalista y se favoreció la influencia de actores conservadores en las decisiones de Estado ${ }^{3}$. A partir de tales ante- cedentes, Chile actualmente no cuenta con un marco legislativo que aborde la especificidad de la salud sexual y reproductiva. Han existido iniciativas estatales, pero no existe un cuerpo legal coherente que siente las bases jurídico institucionales para la formulación e implementación de políticas públicas que 
garanticen los DDSSRR (Hurtado, et al., 2004; Observatorio Equidad de Género en Salud, 2009; ICMER, 2010; Dides y Fernández, 2016).

Muchas veces las iniciativas gubernamentales en el ámbito de la sexualidad y la reproducción han quedado a la voluntad política del momento, dando origen a una política inestable y que no siempre garantiza el ejercicio de los DDSSRR plasmados en los acuerdos firmados y ratificados por Chile (p.ej. El Cairo, 1994; Beijing, 1995). Por tales motivos, interesa analizar la -que podríamos llamar-política de salud sexual y reproductiva en Chile, a partir de las iniciativas impulsada, desarrolladas y frenadas en los últimos cuatro gobiernos (2000-2018). Este ejercicio analítico es interesante considerando los vaivenes de la política en cuestión y la relativamente reciente aprobación de la Ley N²1.030/2017, que regula la interrupción voluntaria del embarazo en tres causales: peligro para la vida de la mujer, inviabilidad fetal de carácter letal y embarazo producto de una violación. Si bien dicha ley representa un avance en la materia, fue una iniciativa largamente discutida, no exenta de polémicas y ante la cual el actual Presidente, Sebastián Piñera (2018-2022), y su bancada se han opuesto y manifestado abiertamente sus intenciones modificarla.

La intención del artículo es comparar los marcos de interpretación ${ }^{4}$ que han orientado el accionar del Estado en cada periodo presidencial y dilucidar énfasis, similitudes y discontinuidades, destacando la contribución de cada mandato en la garantía de los DDSSRR desde una perspectiva de género. El análisis expuesto se nutre de un proceso metodológico que contempló la reconstrucción del proceso de la política (policy tracing) entre los años 2000 y 2018, del análisis a documentos políticos de interés -a través de un critical frame analysisy la aplicación de entrevistas a 39 actores gubernamentales y de la sociedad civil ${ }^{5}$ (Cubillos Almendra, 2014; 2019). Además, se integra información adicional a partir de una revisión documental complementaria (sitios web, periódicos, proyectos de ley, etc.), a modo de profundizar, ampliar y precisar la información proporcionada por las personas entrevistadas.

Con el propósito de exponer las continuidades y las rupturas entre los cuatro gobiernos en el ámbito de la salud sexual y reproductiva, el artículo se ha dividido en tres apartados. El primero describe las prioridades de cada gobierno en el ámbito de política abordado, destacando sus principales iniciativas. El segundo apartado da cuenta de algunas continuidades detectadas en los cuatro mandatos presidenciales. Y, finalmente, la tercera sección concluye retomando las principales ideas abordadas.

\section{Énfasis y prioridades en cada gobierno}

Los cuatro gobiernos, entre el 2000 y el 2018, han presentado matices respecto de su discurso sobre la sexualidad y la inclusión de la sociedad civil en dicho ámbito de política pública. Con la intención de mostrar sus diferentes énfasis, a continuación se trabajan cuatro secciones que ahondan en las prioridades exhibidas por cada mandato presidencial: Ricardo Lagos (2000-2006), Michelle Bachelet (2006-2010), Sebastián Piñera (20102014) y el segundo periodo de Michelle Bachelet (2014-2018).

\section{Ricardo Lagos (2000-2006): la importancia de la participación ciudadana}

El gobierno de Ricardo Lagos (Partido Por la Democracia - PPD ${ }^{6}$ ) estuvo centrado en consolidar la Reforma Sanitaria y un sistema público de protección social. El propósito fue modificar las lógicas impuestas por el régimen militar y sentar las bases de una política de acceso universal sobre criterios de equidad. La Reforma de Salud apuntó a resolver las desigualdades en el ámbito sanitario y fomentar la participación ciudadana, a partir de la institucionalización de mecanismos para ello. Aunque se promovió la equidad, la Reforma Sanitaria no incorporó una perspectiva de género, y la sexualidad y la reproducción no fueron prioridad (Eguiguren, et al., 2009; Matamala, et al., 2011).

Las iniciativas generadas en estos campos, que pueden distinguirse como avances -aunque no libres de polémicas - , fueron las Normas sobre esterilización voluntaria (2000), la Ley sobre VIH/Sida (2001); la inclusión de la anticoncepción de emergencia (AE) en la oferta del sistema de salud público (2001), la prevención y el apoyo al embarazo adolescente, las Normas para la atención a víctimas de violencia sexual (2004), que incluye la AE para las mujeres que han sufrido una violación, y la continuidad de la Mesa Interministerial 
de Prevención del Embarazo Adolescente ${ }^{8}$. Dichas acciones exhibieron una representación de la sexualidad vinculada a un enfoque sanitario-aunque matizado con una perspectiva de derechos humanos y de género-, que se centró principalmente en la regulación de la fertilidad en grupos poblacionales reconocidos como vulnerables (p.ej. jóvenes y mujeres de escasos recursos) (Cubillos Almendra, 2019).

Entre las acciones que impulsaron el diálogo entre el Estado y la sociedad civil en el proceso de la política, adquiere relevancia la conformación de la Mesa de Trabajo en Sexualidad (2002), donde ciertos organismos académicos y ONGs tuvieron oportunidad de discutir y opinar sobre algunas iniciativas, por ejemplo, las Normas para la atención de víctimas de violencia sexual (Fríes, et al., 2007) ${ }^{9}$. Sin embargo, las principales demandas de la sociedad civil fueron desatendidas. Aunque Ricardo Lagos hizo referencia públicamente a la posibilidad de legislar sobre aborto terapéutico, este debate no logró profundidad ni hubo propuestas explícitas en su gobierno ${ }^{10}$. Asimismo, el proyecto de Ley Marco sobre Derechos Sexua- les y Reproductivo (2000), generado por organizaciones de la sociedad civil, no fue discutido en este periodo (Boletín N²608-11).

Una iniciativa interesante fue la política de salud y pueblos originarios (MINSAL, 2005/2006), que buscó ser generada y gestionada con la participación activa de las comunidades indígenas y distintos actores interesados en la temática (expertas/os, implementadores de política y organizaciones sociales). Aunque la formulación de la política no integró explícitamente la sexualidad ni una perspectiva de género, a partir de la demanda en algunas regiones del país (I y IX región) se logra incluir la pertinencia cultural al ámbito de la salud reproductiva (p.ej. parto humanizado), a través del Programa de Salud de la Mujer (MINSAL, 1997) y -posteriormente - el Programa Chile Crece Contigo (2009) ${ }^{11}$.

En lo que respecta a educación sexual, el gobierno de Lagos inició un proceso importante para promover una política nacional, desde una propuesta pedagógica basada en el enfoque derechos humanos, que reconoce la formación en sexualidad como un derecho, sobre criterios de igualdad y no discriminación. No obstante, el Plan de Educación en Afectividad y Sexualidad (MINEDUC, 2005) no fue bien recibido por los sectores ligados a la iglesia católica y no implicó el desarrollo de medidas concretas, lo que redujo sus posibilidades de impacto $^{12}$. El énfasis de este gobierno siguió principalmente la línea de la regulación de la fertilidad en la población adolescente, que intentó modificar las dinámicas tradicionales de la atención en salud, al posicionar a las personas como sujeto de derecho. Sin embargo, este discurso no permeó otros temas vinculados a la sexualidad (p.ej. el debate sobre aborto o el reconocimiento de la sexualidad en otros colectivos).

De este modo, el gobierno de Ricardo Lagos, aunque intentó avanzar en la incorporación de un discurso de derechos humanos en la política pública y motivar mecanismos de diálogo entre Estado y sociedad civil,no integra las demandas y propuestas ligadas a la garantía de los DDSSRR, especialmente el proyecto de ley Marco sobre DDSSRR. Asimismo, la representación social de la sexualidad estuvo más cercana a un enfoque sanitario - que representa la sexualidad ligada principalmente a la reproducción-, que a un accionar del Estado interesado en garantizar los DDSSRR desde una perspectiva integral. Finalmente, cabe destacar las señales que da este gobierno para integrar a nuevos sujetos al espectro de la política, como los pueblos originarios (MINSAL, 2005/2006) y las diversidades sexuales (MINEDUC, 2005).

\section{Michelle Bachelet (2006-2010): énfasis en la igualdad de género}

El primer mandato de Michelle Bachelet (Partido Socialista - PS) siguió la línea trazada por Ricardo Lagos en la inclusión de un enfoque de derechos en las políticas públicas y en el fortalecimiento de la participación ciudadana. No obstante, el énfasis de este gobierno estuvo en la introducción de mecanismos para la transversalización de la perspectiva de género en la gestión del Estado, propósito que estuvo presente en el gobierno anterior -siendo Bachelet Ministra ${ }^{13}$ - , pero que en este periodo toma mayor protagonismo, avanzándose en la aplicación de medidas técnicas concretas (p.ej. Consejos de Género ${ }^{14}$ y compromisos en cada ministerio, y la Agenda de Género). Esta intención presidencial fue alentada, además, por parte del movimiento feminista y de mujeres que se implicó en su campaña y su posterior mandato. 
Durante el gobierno de Lagos, se habló explícitamente sobre garantías universales en salud y es en el gobierno de Bachelet donde toma fuerza la proclama por DDSSRR, que es una demanda del movimiento feminista y de mujeres desde los años 90. Este gobierno mostró sensibilidad ante el tema y generó ciertos avances, desde una lógica de derechos e igualdad de género (MINSAL, 2007). Sin embargo, en opinión de algunas entrevistadas $^{15}$, no se abordaron asuntos claves, como el aborto ${ }^{16} \mathrm{y}$ el proyecto de ley Marco sobre DDSSRR, que se ingresa nuevamente al Parlamento el 2008 (Boletín N5933-11). La Presidenta se comprometió a acelerar el proceso del proyecto de ley en el Parlamento, pero finalmente desistió de hacerlo ${ }^{17}$.

Como el gobierno de Lagos, el primer mandato de Bachelet no propone una política coherente y conun foco claro en el ámbito de la sexualidad y la reproducción, sino más bien iniciativas aisladas enfocadas especialmente en la regulación de la fertilidad de sectores poblacionales reconocidos como vulnerables (especialmente mujeres y jóvenes).Sin embargo, pueden reconocerse ciertos hitos, como las Normas Nacionales de Regulación de la Fertilidad (MINSAL, 2007), que integran explícitamente un enfoque de género, son enunciados desde una lógica de DDSSRR y buscan responder a las demandas de la sociedad civil. Entre otras cosas, dichas Normas plantean la provisión gratuita y universal de la AE a mujeres mayores de 14 años, lo que generó una polémica dado el rechazo que tuvo desde los sectores conservadores. Frente a ello, el Ejecutivo presentó en calidad de suma urgencia un proyecto de ley (2009) que deriva en la Ley N²0.418/2010, que Fija Normas sobre Información, Orientación y Prestaciones en Materia de Regulación de la Fertilidad.

Para algunas entrevistadas ${ }^{18}$ no se observan mayores diferencias entre los gobiernos de Lagos y Bachelet, pues ninguno mostró real interés por abordar estos asuntos ni por incorporar las propuestas emanadas desde la sociedad civil ${ }^{19}$. No obstante, poco antes de dejar la presidencia, Michelle Bachelet dio algunas señales, principalmente a partir del Plan de Igualdad de Oportunidades 2010-2020, que plantea el abordaje de los DDSSRR y la despenalización del aborto terapéutico. Esta intención fue retomada en su segundo mandato.

Respecto a la educación sexual, este gobierno continuó con el Plan Nacional elaborado en el periodo anterior (MINEDUC, 2005). Se buscó incorporar una perspectiva de género y promover la corresponsabilidad en la educación sexual, a partir de la Secretaría Técnica de Educación en Sexualidad, Afectividad y Género ${ }^{20}$ (MINEDUC). Sin embargo -de acuerdo a información proporcionada por profesionales del ministerio y del Programa de Aprendizaje, Sexualidad y Afectividad, de la Universidad de Chile—, dicha Secretaría pareció ser más una declaración de intenciones que una instancia potenciada técnicamente. La implementación del Plan Nacional sólo contempló los programas de Aprendizaje, Sexualidad y Afectividad (PASA) y de Planificación de Educación en Sexualidad y Afectividad. (PLANESA), que apuntaron a la capacitación docente y tuvieron una cobertura acotada.

Por otro lado, este gobierno impulsó el Consejo de Salud de Género (2007) y el Consejo de Jóvenes y Adolescentes (2010), donde se abordaron temas de interés para las organizaciones participantes (p.ej. actualización del Programa de Salud Adolescente y revisión de las Normas de Regulación de la Fertilidad, respectivamente $)^{21}$. No obstante, de acuerdo a lo manifestado por las personas entrevistadas que participaron de ambas instancias, en los Consejos se trataron temas puntuales y que venían predefinidos desde el gobierno. Las propuestas y demandas desde la sociedad civil no fueron incluidas en la agenda pública. Entre tales iniciativas se encuentran el manual pedagógico sobre educación sexual difundido por el Movimiento de Integración y Liberación Homosexual (MOVILH, 2009/2010), la Ley Marco sobre DDSSRR (Boletín N5933-11) y la despenalización del aborto ${ }^{22}$.

Al igual que el gobierno de Lagos, el primer mandato de Michelle Bachelet propone una representación de la sexualidad principalmente desde un discurso sanitario, que vincula la sexualidad a la fertilidad y apunta a su regulación en poblaciones asumidas como vulnerables (mujeres y adolescentes). A pesar que se manifestó públicamente la intención de integrar un discurso de derechos humanos y una perspectiva de género en las iniciativas impulsadas, costó (y ha costado) superar las lógicas biosanitarias para pensar en los DDSSRR de manera integral ${ }^{23}$. No obstante, se reconoce el esfuerzo técnico por transversalizar la perspectiva de género en la gestión del Estado, lo que permitió avances en materia de salud sexual y reproductiva. Por otro lado, si bien se potenciaron los Consejo Consultivo de Salud, siguieron postergándose demandas y propuestas claves desde la sociedad civil. Finalmente, al igual que el gobierno de Lagos, el primer mandato de Michelle Bachelet 
promovió iniciativas que buscaron integrar nuevos beneficiarios al espectro de la política, por ejemplo, al posicionar con mayor énfasis la corresponsabilidad en materia de anticoncepción (MINSAL, 2007).

\section{Sebastián Piñera (2010-2014): el fomento de la maternidad}

El gobierno de Sebastián Piñera (Partido Renovación Nacional - $\mathrm{RN}^{24}$ ) implicó un cambio en las iniciativas estatales llevadas hasta el momento. Gran parte de las propuestas de los dos mandatos anteriores fueron interrumpidas o pierden prioridad. Por ejemplo, el programa de salud y pueblos indígena (MINSAL, 2005/ 2006), los Consejos Consultivos en Salud y la Ley $\mathrm{N}^{\circ} 20.418 / 2010^{25}$. Si se observa cierta continuidad entre los gobiernos de Lagos y Bachelet, este periodo presidencial representa una ruptura significativa con el trabajo desarrollado hasta entonces ${ }^{26}$.

El énfasis del gobierno de Piñera estuvo en el fomento de la familia y la maternidad, lo que limitó la inclusión de una perspectiva de género y la representación de un sujeto de derecho en la política. Evidencia de esto, es que se redujo la distribución de preservativos en la población juvenil ${ }^{27}$ y se desarrollaron programas de fomento a la maternidad (p.ej. Programa de Apoyo a la Maternidad, Programa piloto de fertilidad asistida, 2011). Aunque la arquitectura formal de las políticas de igualdad de género -generada en gobiernos anteriores - se mantuvo, los discursos más conservadores sobre el rol de la mujer en la sociedad permearon las políticas del Servicio Nacional de la Mujer (SERNAM) y otros organismos públicos (Valdés, 2013).

La Ley N²0.418/2010, que fija las normas sobre información, orientación y prestaciones en materia de regulación de la fertilidad, en este gobierno no fue trabajada íntegramente. Se generó un reglamento desde el MINSAL (Decreto $N^{\circ} 48 / 2013$ ), pero no uno desde MINEDUC sobre la formación en sexualidad en establecimientos de educación secundaria (Dides y Fernández, 2016). En cambio, se propusieron siete programas (2011) que - de acuerdo a profesionales MINEDUC - ignoraron el Plan vigente (MINEDUC, 2005). El enfoque de derechos humanos -y con ello el principio de no discriminación e igualdad de género- pierde fuerza, mientras se profundiza en el principio de libertad de enseñanza y el rol subsidiario del Estado. Bajo un argumento de pluralismo, se propusieron programas que no siguieron los planteamientos de la política nacional e instalaron un marcado discurso conservador (Palma, Reyes y Moreno, 2013). De hecho, algunos programas transgredían derechos y garantías definidas en el marco del Plan, al patologizar la homosexualidad (p.ej. Programa Aprendiendo a Querer, Universidad Católica de la Santísima Concepción).

Los mecanismos de diálogo entre Estado y sociedad civil en el gobierno de Piñera fueron más bien nominales. Algunas profesionales de servicios público relacionados a la política estudiada (INJUV, MINEDUC, MINSAL y SERNAM) comentan que durante este gobierno la participación ciudadana decayó, pues se le dio escaso énfasis. Existieron mesas de diálogo, pero no siempre tuvieron continuidad ni lograron incidencia en la formulación de la política. Los Consejos Consultivos en Salud, por ejemplo, siguieron funcionando, pero perdieron importancia en relación a los gobiernos anteriores. Además, los programas de educación sexual propuestos fueron definidos por el gabinete del MINEDUC y ni los profesionales de dicho ministerio tuvieron injerencia ${ }^{28}$.

Los esfuerzos de los gobiernos anteriores por avanzar en términos de participación ciudadana fueron frenados en este periodo presidencial, al promoverse escasamente los espacios de diálogo existentes y al no considerar a las organizaciones de la sociedad civil en la formulación de propuestas. Además, la representación de la sexualidad se vincula a un discurso conservador -ligado a la promoción de los valores de la vida y la familia-, en desmedro de un discurso de derechos humanos (Cubillos Almendra, 2019). Finalmente, este gobierno refuerza la focalización de la política en mujeres heterosexuales en edad fértil -representadas necesariamente como madres-, lo que plantea una ruptura con la intención de los gobiernos anteriores por diversificar e integrar a nuevos usuarios a los beneficios de la política.

\section{Michelle Bachelet 2.0 (2014-2018): los derechos sexuales y reproductivos}

El segundo gobierno de Michelle Bachelet manifiesta explícitamente la necesidad de seguir avanzado en el campo de la igualdad de género y los DDSSRR. Por ejemplo, se creó el Área del buen vivir de la sexualidad 
en SERNAM y el programa del mismo nombre ${ }^{29}$, se presentó (2015) y aprobó (2017) el proyecto de ley sobre interrupción del embarazo en tres causales (Ley $\left.\mathrm{N}^{\circ} 20.030 / 2017\right)^{30}$, se actualizaron las Normas Nacionales de Regulación de la Fertilidad (MINSAL, 2018a) y publica la primera Política Nacional de Salud Sexual y Reproductiva (MINSAL, 2018b). A esto se suma la creación del Ministerio de la Mujer y la Equidad de Género (2016). Estas iniciativas implican necesariamente un cambio de lógicas respecto del gobierno anterior. Se reconoce como un hito que, desde el Ejecutivo, se haya expresado la voluntad de legislar sobre DDSSRR y de incorporar profesionales, vinculadas al movimiento feminista, a la administración estatal ${ }^{31}$ (p.ej. la Ministra y la Subsecretaria de SERNAM, y la encargada de la Unidad de Género en MINSAL) ${ }^{32}$. Al respecto, una entrevistada vinculada al Instituto Chileno de Medicina Reproductiva (ICMER) plantea: "Es histórico, porque nunca un Presidente ha propuesto legislar sobre el tema. Todos han dicho que no van a legislar y lo han declarado en sus campañas presidenciales y durante todos sus gobiernos, y lo repiten sus ministros".

En contraste, otras entrevistadas ${ }^{33}$ se mostraron más escépticas por lo visto el mandato anterior de Michelle Bachelet, o porque se evidenció-durante su primer gobierno - que las demandas de la sociedad civil siguieron siendo postergadas. Por ejemplo, se descartó discutir sobre el Proyecto de Ley Marco sobre DDSSRR. Aunque es destacable la promulgación de la Ley $\mathrm{N}^{\circ} 21.030 / 2017$-sobre interrupción voluntaria del embarazo en tres causales - , que responde, si bien parcialmente, a una demanda histórica del movimiento feminista y de mujeres.

El segundo gobierno de Michelle Bachelet planteó fomentar las instancias de diálogo entre el Estado y la sociedad civil (p.ej. Consejos Consultivos en Salud y mecanismos definidos por la Ley N²0.500/2011) e integrar a diversos actores en la formulación de una política sobre DDSSRR. La propuesta se dirigió a ampliar el foco de la política, no sólo centrada en mujeres en edad fértil, sino en otros sectores usualmente ignorados en el ámbito de la salud sexual y reproductiva (mujeres adultas mayores y hombres adolescentes), desde una lógica de derechos humanos y ciclo vital (p.ej. Programa el Buen Vivir de la Sexualidad y la Reproducción, 2014) ${ }^{34}$. Asimismo, se plantea explícitamente una intención de representar la sexualidad desde un discurso de derechos humanos y una perspectiva de género, al hablar abiertamente de DDSSRR e impulsar iniciativas no sólo vinculadas a la reproducción. Aunque sigue existiendo un fuerte énfasis sanitario, dicha mirada parece ceder espacio ante un discurso de derechos, al intentar promover iniciativas que respeten las decisiones particulares de las personas. Asimismo, se observa un interés por fortalecer los mecanismos de participación ciudadana existentes, a la vez que se propician otros. Finalmente, este gobierno intentó integrar nuevos beneficiarios al ámbito de la política y no centrarse sólo en mujeres en edad fértil. Esto representa un cambio significativo respecto a los gobiernos anteriores - en el abordaje de la sexualidad.

\section{Percepción de continuidades}

Como se ha revisado anteriormente, existen diferencias de énfasis en cada uno de los últimos cuatro gobiernos en materia de sexualidad. No obstante, es posible visualizar ciertas continuidades. Hay quienes consideran que son muchas más las similitudes que las diferencias entre periodos presidenciales, donde ha primado la figura de un Estado ausente y temeroso de abordar la sexualidad en el espacio público. A continuación se ahonda en las semejanzas más relevantes exhibidas entre los años 2000 y 2018, de acuerdo a la opinión de las personas entrevistadas y el material revisado. El apartado se divide en tres secciones que identifican tres elementos de continuidad entre los gobiernos, a saber: la escasa voluntad política y la oposición desde sectores conservadores; la percepción de avances y retrocesos; y la existencia de trabas a nivel institucional y constitucional.

\section{La escasa voluntad política y la oposición de actores conservadores}

En opinión de las personas entrevistadas, mayoritariamente pertenecientes a organizaciones de la sociedad civil ${ }^{35}$, una de las principales similitudes entre los cuatro gobiernos se refiere a la escasa voluntad política de abordar la sexualidad como un asunto público, dadas por las resistencias desde sectores conservadores. La sexualidad y la reproducción, hasta el momento, han sido abordadas principalmente desde un discurso sanitario, que ha tendido a ceder a las presiones desde los sectores conservadores (iglesias cristianas, partidos conser- 
vadores ${ }^{36}$ y organizaciones “pro-vida”). En ninguno de los cuatro gobiernos se visualizaría una intención concreta de generar una política coherente, con directrices claras, que contemple los diversos elementos que implica pensar la sexualidad de modo integral y reconozca la autonomía de las personas. El gobierno más próximo a esto fue el segundo mandato de Michelle Bachelet.

La sexualidad es sindicada como un asunto que genera bastantes rencillas y reticencias políticas, por lo que nunca ha sido prioridad gubernamental ni se han emprendido mayores esfuerzos por formular una política integral, intersectorial y con la participación de los diversos sectores de interés. Con ello, se evade el tema de fondo y sólo se generan iniciativas reactivas y/o parciales ante contingencias o polémicas específicas (p.ej. niñas abusadas sexualmente y embarazadas producto de ello o las polémicas en establecimientos educaciona- les). Estas acciones, además, no tratan la sexualidad en sentido positivo y no garantizan la autonomía de las personas.

Han existido reacomodos en la política a nivel nacional (p.ej. las Normas Nacionales de Regulación de la Fertilidad, la Ley sobre interrupción del embarazo en tres casuales, la distribución de la AE y algunos programas de educación sexual), pero no la formulación de una política coherente que garantice el ejercicio autónomo de la sexualidad, en concordancia con los DDSSRR y los acuerdos firmados y ratificados por el Estado chileno. Pareciera que sólo se han generado iniciativas con el fin de dejar tranquila a la opinión pública, donde además los sectores conservadores suelen tener amplia influencia, por lo que no se han propuesto medidas satisfactorias ni robustas. Prueba de esto es la educación sexual, existe un Plan Nacional (MINEDUC, 2005), la Ley N²0.418/ 2010 proclama su obligatoriedad en la educación secundaria, se han desarrollado manuales (guías, orientaciones) y se han implementado algunos programas, pero estas acciones son insuficientes para instalar una política nacional que se precie de tal. No se han desarrollado evaluaciones, la cobertura no ha sido satisfactoria y los establecimientos educativos gozan de la autonomía para definir qué recomendaciones de MINEDUC tomar y cuáles desatender ${ }^{37}$. No existe una propuesta de Estado clara, exigible ni garantizable. Son las crisis o polémicas concretas las que detonan el debate público y en varias oportunidades ni eso es suficiente para producir cambios significativos ${ }^{38}$.

Una posible explicación de la escasa voluntad exhibida por los cuatro gobiernos alude a los costos políticos asociados de tratar la sexualidad como tema público (Shepard, 2000; Pecheny y de la Dehesa, 2011). Los gobiernos serían reticentes a tratar abiertamente estas materias desde una lógica más progresista, por los posibles conflictos que esto pueda generar al interior de las coaliciones políticas (p.ej. la Nueva Mayoría ${ }^{39}$ ) y con los sectores conservadores. Lo que, además, podría repercutir en el porcentaje de aprobación por parte de la opinión pública y/o actores políticos influyentes. Un ejemplo de esto fue la polémica sobre el aborto y la renuncia de la ex Ministra de Salud, Helia Molina (2014). Molina manifestó -en una entrevista a un periódico nacional $^{40}$ - , que una política restrictiva como la chilena genera que quienes tienen los recursos económicos puedan acceder al sistema de salud privado para interrumpir sus embarazos. Estas declaraciones fueron ampliamente criticadas por los sectores conservadores y derivaron en la renuncia de la entonces ministra.

Los costos políticos perpetúan la marginalidad de quienes defiendes los DDSSRR y permiten tener tras bambalinas los desacuerdos entre actores (Shepard, 2000, p. 137). Por estas razones, los gobiernos se han cuidado de no activar fricciones en el debate y moverse con extrema cautela respecto de las iniciativas que emprenden y/o las declaraciones públicas que realizan. El movimiento feminista y las organizaciones de la sociedad civil han contribuido a hacer frente a estos costos políticos, prestando apoyo a quienes se manifiestan a favor de estas demandas y visibilizando el doble discurso (Shepard, 2000) que da origen a políticas públicas restrictivas. Además, las organizaciones feministas y de mujeres han buscado generar presiones constantes para ampliar la discusión y visibilizar las demandas y necesidades de la población, más allá de una política de consensos entre actores gubernamentales. Sin embargo, estas intenciones no siempre calan en el debate público ni las decisiones gubernamentales, y las iniciativas siguen una lenta trayectoria hasta diluirse en el tiempo.

\section{La percepción de avances y retrocesos}

Ligado a la escasa voluntad política de los gobiernos y la influencia de sectores conservadores, se encuentra la percepción generalizada de que en el ámbito de la sexualidad se observan avances lentos (o "avan- 
ces-retrocesos" ${ }^{41}$. Esto ocurriría dado que, al momento de reportarse algunos avances, prontamente viene aparejado un retroceso; o se trata de un avance débil. Ejemplo de ello son las Normas Nacionales de Regulación de la Fertilidad, que permite, entre otras cosas, la distribución de la AE en el sistema de salud público (incluidas mujeres de menos de 14 años). No obstante, esta medida fue ampliamente cuestionada y frenada por sectores conservadores (p.ej. no distribuida en municipios de gobiernos opositores/conservadores), lo que relativiza este avance. Algo similar ha pasado con la Ley $\mathrm{N}^{\circ} 20.418 / 2010$, que representa una mejora en materia legislati- va respecto al acceso a información sobre regulación de la fertilidad y a educación sexual, pero el proceso para validar un reglamento desde el MINEDUC ha sido lento. Existe la ley, pero no se crean oportunamente mecanismos que permitan su aplicación.

El debate sobre la sexualidad parece estancado. Se movería en lo mismo: los sectores conservadores continúan teniendo un rol preponderante y los gobiernos no quieren (re)activar polémicas. Esto ha llevado a proponer iniciativas "matizadas", que no satisfacen del todo a ninguno de los grupos de interés y permiten la aplicación acomodadiza de la política. Por verbigracia podría mencionarse la timidez política que ha caracterizado a los gobiernos frente a la educación sexual y el aborto. En ninguno de estos dos asuntos los gobiernos se han mostrado abiertamente contrarios a los planteamientos de sectores conservadores, sino más bien han buscado acomodar su discurso con el propósito de satisfacer las demandas de diversos actores, aun cuando son discordantes.

En educación sexual se ha generado un Plan Nacional desde lógicas de derechos humanos, nodiscriminación e igualdad, y a la vez se ha respetado la libertad de enseñanza y un rol subsidiario del Estado. Este planteamiento "matizado" produce contradicciones que aún no han sido resueltas y limita que los programas, en su aplicación, definan mínimos exigibles que respeten los principios proclamados por la política ${ }^{42}$. En relación al aborto, no es hasta el segundo gobierno de Michelle Bachelet donde el Ejecutivo se muestra a favor de despenalizarlo por razones terapéuticas, a partir de la insistencia desde organizaciones de la sociedad civil. Empero, este asunto se trató con cautela, con el fin de no despertar fricciones políticas ${ }^{43}$.

Otro aspecto que influye en esta percepción de "avances-retrocesos" es la escasa continuidad entre los gobiernos, donde se observan iniciativas más bien intermitentes -en términos de presupuesto, profesionales y énfasis - y donde cuatro años parecen ser pocos para consolidar ciertas iniciativas ${ }^{44}$. Ejemplo de esto es el Programa de Salud Adolescente (MINSAL, 2012) que, de acuerdo lo que comenta una profesional SERNAM, ha tenido altos y bajos. En ocasiones se le ha dado prioridad y presupuesto pertinente, y otras se ha subordinado a la política dirigida a las mujeres (MINSAL, 1997).

Algo similar ha sucedido con el Programa de Salud y Pueblos Indígenas (MINSAL, 2005/2006) y los Consejos Consultivos en Salud, los que durante el gobierno de Piñera no tuvieron prioridad ${ }^{45}$. Ejemplos similares pueden hallarse en el ámbito de la educación sexual, donde a pesar de existir un Plan Nacional no ha habido continuidad en los programas y no se observan avances sustantivos desde el gobierno de Ricardo La$\operatorname{gos}^{46}$. Frente a esto, queda la sensación de que no existe una voluntad genuina de avanzar en la garantía de DDSSRR. Por otro lado, pueden hallarse obstáculos más difíciles de sortear, que aluden a los establecidos a nivel constitucional, que imponen una forma de hacer política pública que no permite transformaciones sustantivas. En la siguiente sección se aborda este aspecto.

\section{Trabas a nivel constitucional: la necesidad de reformar la Constitución}

El modo de formular políticas públicas en los cuatro gobiernos no se ha modificado, lo que en parte concierne a las restricciones que establece la actual Constitución (1980) ${ }^{47}$. La Constitución -que promueve un modelo neoliberal, autoritario y ultraconservador - fue elaborada de modo que el arco de acción política, independiente de quien gobierne, no permite modificar la competencia electoral bipactista instalada por la dictadura militar (Garretón y Garretón, 2010).

La Constitución de 1980 consagró la exclusión política a través del sistema binominal a nivel parlamentario, que impone límites a la democracia electoral, dadas sus restricciones fácticas y normativas (Siavelis, 2004; Altman, 2004; Rubano, 2007). Asimismo, "la dimensión ciudadana de la democracia se enfrenta a pro- 
blemas de derechos civiles específicos de las minorías, desigualdades socioeconómicas, participación electoral y ausencia de instituciones de participación" (Garretón y Garretón, 2010, p.146). Al ser la única Constitución en el mundo adoptada en una dictadura que continúa vigente en un régimen democrático, no existe consenso respecto de su legitimidad ni si representa la voluntad de la sociedad chilena (Garretón y Garretón, 2010). Frente a esto es amplia la demanda por un cambio constitucional, ya no sólo a partir de reformas que modifi- quen fracciones de ésta, sino una nueva Constitución que emane de una Asamblea Nacional Constituyente (AC) $)^{48}$.

Entre las/os entrevistadas/os ${ }^{49}$ se reconoce que uno de los principales problemas, para que la legislación sobre salud sexual y reproductiva en Chile progrese, fue el sistema binominal a nivel parlamentario propuesta por la Constitución de 1980. El sistema binominal -actualmente modificado por la Ley N²0.840/2015- aplicaba la lógica de mayoría relativa que instauró un sistema bipartidista en las elecciones legislativas. Esto aseguraba la participación del bloque conservador en el Parlamento, que anterior a la Constitución de 1980 se presentaba como minoritario, generando su sobre representación (Siavelis, 2004). Fue en el Parlamento donde se encontraron las mayores trabas para, por ejemplo, despenalizar la interrupción voluntaria del embarazo - uno de los temas más polémicos en materia de salud sexual y reproductiva en Chile- o instalar una Ley que garantice y proteja los DDSSRR.

El caso específico de la despenalización del aborto (no sólo en tres causales) es más complejo, ya que requiere de un quórum parlamentario de dos terceras partes de las/os diputadas/os y las/os senadoras/es en ejercicio (Art. 127), dado que la Carta Fundamental protege la vida del que está por nacer (Art. 19). El alto quórum parlamentario para el desarrollo de este tipo de modificaciones legislativas y el sistema binominal (vigente hasta mayo 2015) han sido reconocidas - por las personas entrevistadas - como unas de las principales razones por las que se apela a una reforma constitucional para avanzar en materia de DDSSRR. En este sentido, la Ley $\mathrm{N}^{\circ} 20.840 / 2015$, que sustituye el sistema binominal por uno de carácter proporcional (Método D'Hondt) en la conformación del Congreso Nacional, podría ser un eventual avance para legislar sobre DDSSRR. No obstante, sigue siendo pertinente una reforma constitucional (Corporación Humanas, 2016). Al respecto, una integrante de Corporación Humanas expresa:

“en Chile las instituciones y el marco normativo fundamental - que es la Constitución política - está en cuestión y una nueva Constitución para ser legítima, necesariamente, tiene que incorporar los avances en Derechos Humanos que se dan a nivel internacional. Esto implica que los derechos sexuales y reproductivos tienen que ser parte de este pacto constitucional nuevo al que Chile va a arribar y eso es necesario para tener garantía de que estos derechos efectivamente se puedan cumplir."

Adicionalmente, es la misma Constitución la que limitaría una democracia plena, donde la ciudadanía tenga una incidencia real en el cómo se hace política a nivel nacional y cómo se formulan las políticas públicas. Como manifestó el Senador Alejandro Navarro (Partido Movimiento Amplio Social, MAS ${ }^{50}$ ), en la una de las sesiones del Senado donde se discutió la posibilidad de legislar sobre aborto terapéutico:

"Me gustaría que sobre estos asuntos tuviésemos la posibilidad de realizar un plebiscito vinculante, en el cual se pudiera consultar a la ciudadanía sobre un problema en que están implícitos la fe, la moral, el laicismo, la convicción, porque es un tema país. Pero Chile no tiene ese tipo de consulta. La Constitución no brinda la herramienta para poder preguntar a los ciudadanos (Senado de la República de Chile, 2012, p. 121)."

Una de las grandes limitaciones para la formulación de políticas públicas inclusivas y con participación de los diferentes sectores y actores políticos estaría dada por una Constitución que no propicia un contexto político favorable para generar modificaciones en la legislación ${ }^{51}$. La actual Constitución suscitaría lógicas autoritarias e impositivas, lo que limita el concebir políticas que permitan la emergencia de diversos proyectos ético-políticos, que cuenten con la participación e injerencia de diversos actores y grupos de opinión, y consideren las realidades de sujetos diversos y su autonomía.

En opinión de algunas/os entrevistadas/os ${ }^{52}$, la sociedad chilena estaría preparada para realizar cambios 
sustantivos en la Constitución y en el modo de hacer política. Actualmente, hay mayor apertura para entablar ciertos temas en el ámbito público (sexualidad, diversidad sexual, aborto, etc.), y existe mayor consciencia sobre derechos y la capacidad de decidir. La sociedad en Chile estaría experimentando un cambio cultural que le lleva a demandar más del Estado, no sólo políticas paternalistas, sino políticas que reconozcan la pluralidad social existente (distintos sistemas de valores, estilos de vida y modos de vivir la sexualidad) y que conciban agentes críticos, no meros receptores de las políticas públicas. Hay personas y grupos que están dispuestos a posicionar la sexualidad como asunto público, vinculado a la ciudadanía, la autonomía y la autodeterminación, donde el Estado más que un actor que debe regular la sexualidad en base a un ideal normativo (ya sea moral o sanitario), debe permitir la emergencia de diversos proyectos ético-políticos, apoyando y garantizando las deci- siones particulares.

\section{Conclusiones}

En el presente artículo se ha hecho un breve análisis sobre la trayectoria de la política de salud sexual y reproductiva en Chile, a partir de los cuatro gobiernos presentes en el periodo 2000-2018. En primera instancia, se ha reparado en los énfasis de cada mandato presidencial. Las prioridades del gobierno de Ricardo Lagos (2000-2006) estuvieron en la inclusión de un discurso de derechos humanos en las políticas públicas -especialmente en el ámbito de la salud y la protección social—, y en el establecimiento de mecanismos de diálogo entre Estado y sociedad civil. No obstante, la sexualidad no fue prioritaria, aunque se observan avances, principalmente, desde una lógica sanitaria, que se enfoca en la regulación de la fertilidad, la prevención del embarazo adolescente y problemas sanitarios asociados a la reproducción.

El primer gobierno de Michelle Bachelet (2006-2010) continuó el trabajo de Ricardo Lagos sobre la inclusión del enfoque de derechos humanos y el afianzamiento de espacios de diálogo entre Estado y sociedad civil, enfatizando en la introducción de una perspectiva de género en la administración del Estado. La salud sexual y reproductiva adquiere mayor importancia y se visualizan mejoras que buscan favorecer la igualdad de género y la corresponsabilidad en la regulación de la fertilidad. Estas iniciativas, aunque también tuvieron un marcado énfasis sanitario, intentaron ser trabajadas desde una lógica de derechos y equidad de género. Así, tanto el gobierno de Lagos como el primer gobierno de Bachelet se muestran proclives a avanzar en términos de participación ciudadana y derechos humanos.

El gobierno de Sebastián Piñera (2010-2014) representó un cambio importante respeto de los mandatos presidenciales anteriores en materia de salud sexual y reproductiva, igualdad de género y participación social. Las intenciones políticas de este gobierno buscaron promover un discurso conservador, que reforzó la figura de la "mujer-madre" y restó importancia a los mecanismos de diálogo iniciados por los gobiernos anteriores.

El segundo mandato de Michelle Bachelet, por su parte, se mostró más proclive a debatir y emprender acciones en torno a los DDSSRR y la igualdad de género. Hay una intención expresa por avanzar en estas materias desde una lógica de derechos, igualdad y no discriminación. En términos comparativos, este gobierno es el que se ha mostrado más proactivo en el ámbito de política estudiado, buscando profundizar las estructuras de igualdad de género, integrar a profesionales y activistas feministas a la administración del Estado, introducir a su programa algunas de las demandas del movimiento feminista y fortalecer el diálogo entre el Estado y la sociedad civil.

Si bien se corrobora en cierta medida la hipótesis planteada -respecto que no ha existido voluntad política suficiente por parte de los gobiernos entre 2000 y 2018 para abordar integralmente el ámbito de la sexualidad, en concordancia con los DDSSRR y una perspectiva de género-, cabe considerar que los dos gobiernos de Bachelet abren ventanas de oportunidad política (Kingdon, 1984) para este cometido, aunque no pueda afirmarse que se ha avanzado de modo enteramente satisfactorio. Aún hay demandas desde los movimientos sociales que han quedado al margen en la agenda pública y sobre los que cabría seguir avanzando.

Las dificultades para proponer una política a la luz de los DDSSRR, no sólo estarían determinadas por la voluntad política de cada gobierno, sino también por obstáculos dados por la institucionalidad del Estado y 
la actual Constitución (1980). En este sentido, las similitudes de los cuatro gobiernos darían cuenta de las principales restricciones para concretar una política que garantice los DDSSRR y que responda a las demandas de pluralismo generadas desde la sociedad civil. Entre estas dificultades se observan principalmente dos: i) una escasa voluntad política desde los gobiernos frente a las presiones generadas desde sectores conservadores, lo que han limitado el posicionamiento de la sexualidad como tema de interés público y ha dado pie a avances parciales; y ii) una Constitución Política, herencia de la dictadura cívico-militar, que ha impedido generar cambios sustantivos en el cómo se formulan y gestionan las políticas públicas a nivel nacional.

Entre los principales obstáculos impuesto por la Constitución vigente pueden identificarse las limitaciones que esta misma impone para modificarla, las dinámicas jerárquicas de interlocución entre el Estado y la sociedad civil que propone y la no garantía de los DDSSRR de manera explícita. A pesar de las modificaciones introducidas desde los años 90, la toma de decisiones sobre las políticas públicas aún parece un espacio burocrático impenetrable que no necesariamente facilita ni valora el diálogo político entre diversos actores. La participación de la sociedad civil parece ser representada más bien como una intención accesoria, que no implica un cambio sustantivo en el modo de formular y gestionar las políticas públicas. El Estado y la sociedad civil, así, difícilmente entran en sintonía y coordinación colaborativa, lo que restringe la incorporación de diferentes sistemas de valores, demandas y necesidades, a la vez que no reconoce el ejercicio de autonomía de los y las ciudadanas, ni se garantizan sus DDSSRR.

\section{Agradecimientos}

Beca Programa de Formación de Capital Humano Avanzado. Comisión Nacional de Investigación Científica y Tecnológica (Chile). CONICYT BCH/Doctorado Extranjero/2012-72130025.

\section{Notas}

${ }^{1}$ Se entiende por política de salud sexual y reproductiva todas aquellas acciones (e inacciones) gubernamentales que abordan el campo de
la sexualidad y la reproducción. Para el análisis se han priorizado la educación sexual y la regulación de la fertilidad por ser ámbitos polémicos en Chile.

${ }^{2}$ Esta perspectiva reconoce y es crítica del sistema sexo/género, que otorga significados, valores, normas, prácticas sociales a los sujetos en base a su diferencia sexual anátomo-fisiológica. Dicho sistema ha dado sentido a las relaciones sociales que han (re)producido y naturalizado los roles sociales asumidos para hombres y mujeres, la supremacía masculina sobre las mujeres (y "lo femenino") y la heterosexualidad (Rubin, 1975, 1984; Barbieri, 1993).

${ }^{3}$ Desde los sectores conservadores se han opuesto, por ejemplo, a incluir programas de educación sexual en el currículum escolar, garantizar la distribución universal de la anticoncepción de emergencia (AE), y despenalizar la interrupción voluntaria del embarazo en tres causales (Jacobs, 2004; Dides, 2004; Dides, et al., 2007; Guzmán y Seibert, 2010; Dides, et al., 2013; Dides y Fernández, 2016).

${ }^{4}$ Para mayor información sobre los marcos de interpretación presentes en el debate sobre la salud sexual y reproductiva en Chile, véase Cubillos Almendra, 2019.

${ }^{5}$ Se entrevistaron 14 profesionales de organizaciones gubernamentales, 8 representantes de organismos académicos y 17 representantes de organizaciones sociales y ONGs - entre los meses de junio y octubre 2014 , quienes se han interesado en el debate sobre la salud sexual y reproductiva en Chile.

${ }^{6}$ Partido político de centro-izquierda.

${ }^{7}$ Frente al interés del gobierno de Lagos, desde la Organización Panamericana de la Salud (OPS) y el movimiento feminista se inicia un trabajo con la Universidad de Chile y el Servicio Nacional de la Mujer (SERNAM) que derivó en una propuesta sobre la inclusión de la perspectiva de género en la Reforma de Salud. No obstante, el gobierno de Lagos no incluyó las recomendaciones. A partir de esta iniciativa, el 2003 se crea el Observatorio de Equidad en Salud (Región Metropolitana) y el Observatorio Regional en Salud según Género y Pueblo Mapuche (ORSGPM, IX Región). Entrevista integrante de la Articulación Feminista por la Libertad de Decidir (AFLD) y ex profesional del Ministerio de Salud (MINSAL).

${ }^{8}$ Esta mesa implementa el Programa Nacional del mismo nombre y busca instalar el tema como política pública en materia de salud sexual y reproductiva para la población juvenil y adolescente, desde un enfoque intersectorial, convocando a los Ministerios de Salud y Educación, el Instituto Nacional de la Juventud (INJUV) y el SERNAM. Cabe mencionar que - de acuerdo a las profesionales de MINSAL, INJUV y SERNAM entrevistadas - el trabajo intersectorial no siempre ha sido posible. 
${ }^{9}$ Entrevistas profesionales Instituto Chileno de Medicina Reproductiva (ICMER) y MINSAL.

${ }^{10}$ Entrevista representante AFLD y ex profesional MINSAL.

${ }^{11}$ Entrevista profesional Oficina de Salud y Pueblos Indígenas en MINSAL.

${ }^{12}$ Entrevistas representantes Movimiento de Integración y Liberación Homosexual (MOVILH) y profesionales Programa de Aprendizaje en Sexualidad, Afectividad y Género (PASA), Universidad de Chile.

${ }^{13}$ Algunas personas entrevistadas reconocen la importancia de la figura de Michelle Bachelet, como Ministra de Salud del gobierno de Lagos, en la incorporación de una perspectiva de género en las iniciativas sanitarias.

${ }^{14}$ Esta propuesta fue generada desde el movimiento feminista y acogida por la Presidenta Bachelet. Cada ministerio contó con una persona a cargo de la inclusión de la perspectiva de género en la gestión ministerial. Aunque esta iniciativa no siempre contó con el presupuesto suficiente. Entrevista integrante AFLD y ex profesional MINSAL.

${ }^{15}$ Entrevistas representantes ICMER, Corporación Humanas, Colectiva La Huacha Feminista y AFLD.

${ }^{16}$ En 2009, el gobierno de Chile rechaza las recomendaciones del Examen Periódico del Consejo de Derechos Humanos de Naciones Unidas sobre el tema.

${ }^{17}$ Entrevista integrante AFLD y ex profesional MINSAL.

${ }^{18}$ Entrevistas representantes Centro de Medicina Reproductiva y Desarrollo Integral del Adolescente (CEMERA), Línea Aborto Libre (LAL), Colectivo Universitario de Disidencia Sexual (CUDS), La Huacha Feminista, Católicas por el Derecho a Decidir (CDD-Chile).

${ }^{19}$ Algunas profesionales y activistas feministas que contribuyeron al trabajo de este gobierno manifestaron descontento, plasmado en el libro Y votamos por ella. Michelle Bachelet: miradas feministas (Burotto y Torres, 2010).

${ }^{20}$ Esta iniciativa se desprende del proceso impulsado por Lagos (2004-2005).

${ }^{21}$ Entrevistas representantes Corporación Humana, MINEDUC y MINSAL.

${ }^{22}$ Entrevistas miembros ICMER, Corporación Humanas, La Huacha Feminista, AFLD y MOVILH.

${ }^{23}$ Entrevistas representantes de ICMER, MINSAL, Corporación Humana, ORSGPM y AFLD.

${ }^{24}$ Partido político de derecha.

${ }^{25}$ Entrevistas profesionales MINSAL, MINEDUC y SERNAM.

${ }^{26}$ Entrevistas representantes INJUV, MINEDUC, Corporación Humana y SERNAM.

${ }^{27}$ Entrevistas profesionales INJUV.

${ }^{28}$ Entrevistas profesionales MINEDUC.

${ }^{29}$ Entrevista profesional SERNAM, encargada del Área y el Programa a nivel nacional.

${ }^{30}$ Esto también implicó la publicación de la Norma técnica sobre acompañamiento integral a las mujeres que se encuentran en alguna de las tres causales que estipula la Ley N²1.030 (2018).

${ }^{31}$ Esto ya había ocurrido en su primer gobierno, pero en el segundo mandato se expresa con mayor énfasis.

${ }^{32}$ Entrevistas representantes ICMER, MINSAL y CDD-Chile.

${ }^{33}$ Entrevistas integrantes LAL, CUDS, La Huacha Feminista y AFLD.

${ }^{34}$ Entrevista profesional SERNAM.

${ }^{35}$ Entrevistas representantes MINEDUC, CEMERA, MOVILH, Corporación Humanas, LAL, CUDS, La Huacha Feminista, Corporación Nacional de Desarrollo Indígena (CONADI), CDD-Chile, AFLD y PASA.

${ }^{36}$ Partidos con orientación política de derecha: Democracia Cristiana (DC), Unión Demócrata Independiente (UDI) y Renovación Nacional (RN).

${ }^{37}$ Entrevista a profesionales MINEDUC y profesionales PASA. 
${ }^{38}$ Entrevista profesionales PASA.

${ }^{39}$ Coalición política de la ex Presidenta Bachelet, donde el partido Demócrata Cristiano ha mostrado una importante influencia.

${ }^{40}$ De Améstica, Catalina (2014, 30 diciembre) "Ministra Molina: 'En todas las clínicas cuicas, muchas familias conservadoras han hecho abortar a sus hijas"”, La Segunda. Recuperado de: http://www.lasegunda.com/Noticias/Naciona1/2014/12/984214/ministra-molina- entodas-las-clinicas-cuicas-muchas-familias-conservadoras-han-hecho-abortar-a-sus-hijas (24-04-2019).

${ }^{41}$ Entrevistas a activista feminista aymara y representantes CEMERA, MINSAL, CONADI y CDD-Chile.

${ }^{42}$ Entrevistas representantes MOVILH y PASA.

${ }^{43}$ Entrevista representante AFLD y ex profesional MINSAL.

${ }^{44}$ Entrevista profesional CEMERA.

${ }^{45}$ Entrevistas representantes MINSAL y Corporación Humanas.

${ }^{46}$ Entrevistas representantes MINEDUC, MOVILH y PASA.

${ }^{47}$ Entrevistas: activista feminista aymara y representantes INJUV, Red de Mujeres Mapuche (Santiago), Colectivo Palos de Ciego, Corporación Humanas y Círculo Emancipador de Mujeres y Niñas con Discapacidad (CIMUNIDIS).

${ }^{48}$ A fines de la primera década del año 2000, se levanta un movimiento ciudadano que demanda la derogación de la actual Constitución. El propósito fue impulsar la conformación de una Asamblea Constituyente que permita la participación democrática en la redacción de una nueva constitución.

${ }^{49}$ Entrevistas representantes INJUV, MINSAL y CDD-Chile.

${ }^{50}$ Partido político de izquierda.

${ }^{51}$ Cabe mencionar que la propuesta de la ex Presidenta Michelle Bachelet sobre formular una nueva Constitución (2015) aún no se ha concretado.

${ }^{52}$ Entrevistas representantes ICMER, MOVILH, Servicio Nacional del Adulto Mayor (SENAMA), LAL, CUDS y SERNAM.

\section{Bibliografía}

Altman, D. (2004). Redibujando el mapa electoral chileno: Incidencia de factores socioeconómicos y género en las urnas. Revista de Ciencia Politica, XXIV (2), 49 - 66. DOI: 10.4067/S0718-090X2004000200003

Barbieri, T. de (1993). Sobre la categoría de género: una introducción teórico-metodológica. Debates en Sociología, 18, 145-169.

Bonan, C. (2004). Derechos sexuales y reproductivos, reflexividad y transformaciones de la modernidad contemporánea. En: Análisis comparado de legislación, políticas públicas e instituciones orientadas hacia el logro de la equidad de género. Buenos Aires, Argentina: PRIGEPP-FLACSO.

Burotto, A., y Torres, C. (2010) (eds.). Y votamos por ella. Michelle Bachelet: miradas feministas. Santiago, Chile: Andros.

Corporación Humana. (2016). Nueva Constitución y Derechos Sexuales y Reproductivos. Santiago, Chile: Corporación Humana

Cubillos Almendra, J. (2014). Reflexiones sobre el proceso de investigación. Una propuesta desde el feminismo decolonial. Athenea Digital. Revista de Pensamiento e Investigación Social, 14(4), 261-285. DOI: $10.5565 / \mathrm{rev} /$ athenea. 1343

Cubillos Almendra, J. (2019). Los marcos interpretativos en la política chilena de salud sexual y reproductiva. Papers. Revista de Sociología, 104(4). DOI:10.5565/rev/papers.2539

Dides, C. (2004). Aproximaciones a los debates públicos sobre sexualidad y reproducción: aprendizajes sobre la 
Polis, Revista Latinoamericana, $N^{\circ} 53$ | 2019

Javiera Cubillos Almendra introducción de anticoncepción de emergencia en Chile. En C. Cáceres, F. Timothy, M. Pecheny y V. Terto (eds.). Ciudadanía Sexual en América Latina: abriendo debate. Lima, Perú: Universidad Peruana Cayetano Heredia.

Dides, C., Márquez, A., Guajardo, A., y Casas, L. (2007). Chile: Panorama de sexualidad y derechos humanos. Santiago, Chile: CLAM.

Dides, C., Nicholls, L., Bozo, N., y Fernández, C. (2013). Provisión de Anticoncepción de Emergencia en el Sistema de Salud Municipal de Chile. Estado de situación 2013. Serie de Documentos No3. Santiago, Chile: Universidad Central.

Dides, C. y Fernández, C. (2016) (eds.). Primer informe salud sexual, salud reproductiva y derechos humanos en Chile. Santiago, Chile: MILES-Chile.

Eguiguren, P., Cavil, M. E., Díaz, X., Ferres, M., Iglesias, M. y Olavarría, J. (2009). Observatorio de Equidad de Género en Salud - Chile: una mirada a las políticas públicas. Rev Chil Salud Pública, 13(2),106-111. DOI: $10.5354 / 0719-5281.2009 .644$

Fríes, L., Lorenzini, K. y Zavala, X. (Coord.) (2007). Informe Sombra CEDAW. Chile 2003-2006. Santiago, Chile: Corporación Humanas.

Garretón, M., y Garretón, R. (2010). La democracia incompleta en Chile: La realidad tras los rankings internacionales. Revista de ciencia politica, 30(1), 115-148. DOI: 10.4067/S0718-090X2010000100007

Guzmán, V., y Seibert, U. (2010). The churches and gender equality in Chile. Religious impact on sex education policies and on the introduction of emergency contraception. Final Research Report prepared for the project Religion, Politics and Gender Equality. UNRISD. Heirich Böll Stiftung.

Hurtado, J., Pérez, S., y Dides, C. (2004). El debate sobre los derechos sexuales y reproductivos en Chile: ¿Separación Iglesia-Estado?. En C. Dides (Comp.). Diálogos Sur-Sur sobre religión, derechos y salud sexual y reproductiva: los casos de Argentina, Colombia, Chile y Perú. Santiago, Chile: UAHC.

ICMER (2010). Marco Conceptual para Politicas y Programas de Salud Sexual y Reproductiva. Instituto Chileno de Medicina Reproductiva.

Jacobs, E. (2004). Sexuality in Chile. Independent Study Project (ISP) Collection. SIT Study Abroad.

Kingdon, J. (1984). Agendas, Alternatives and Public Policies. Boston, USA: Little Brown.

Labarca, C. (2008). 'Todo lo que usted debe saber sobre las enfermedades venéreas'. Las primeras campañas de educación sexual estatales entre 1927 y 1938. En: M. S. Zárate (Comp.) Por la salud del cuerpo. Historia y politicas sanitarias en Chile. Santiago, Chile: Universidad Alberto Hurtado.

Matamala, M.; Eguiguren, P. y Díaz, X. (2011). Tensiones y Silencios en la reforma de la salud. Santiago, Chile: Observatorio de Equidad de Género en Salud.

MINEDUC (2005). Plan Nacional de Educación en Sexualidad y Afectividad. Ministerio de Educación, Chile.

MINSAL (1997). Programa de Salud de la Mujer. Ministerio de Salud, Chile.

MINSAL (2005/2006). Política de salud y pueblos indígenas. Segunda Edición. Ministerio de Salud.

MINSAL (2007). Normas Nacionales de Regulación de la Fertilidad. Ministerio de Salud, Chile. 
MINSAL (2012). Programa Nacional de Salud Integral de Adolescentes y Jóvenes. Plan de Acción 2012-2020. Ministerio de Salud, Chile.

MINSAL (2018a). Normas Nacionales de Regulación de la Fertilidad. Ministerio de Salud, Chile.

MINSAL (2018b). Politica Nacional de Salud Sexual y Salud Reproductiva. Ministerio de Salud, Chile.

MOVILH (2009/2010). Educando en la diversidad. Orientación sexual e identidad de género en las aulas. Segunda Edición. Santiago, Chile: Movimiento de Integración y Liberación Homosexual.

Observatorio Equidad Género en Salud (2009). Informe 2007-2008. Santiago: Universidad de Chile.

Olavarría, J. (2005). La política de educación sexual del Ministerio de Educación de Chile. Consideraciones para una evaluación. Ponencia presentada en el Seminario Internacional: Equidad de Género en las Reformas Educativas de América Latina. Santiago, Chile (17-18 mayo).

Palma, I., Reyes, D., y Moreno, C. (2013). Educación sexual en Chile: Pluralismo y libertad de elección que esconde una propuesta gubernamental conservadora. Docencia, 49, 14-24.

Pecheny, M., y De la Dehesa, R. (2011). Sexualidades y políticas en América Latina: un esbozo para la discusión. En S. Corrêa, y R. Parker (Org.). Sexualidade e política na América Latina: históricas, interseções e paradoxos. Rio de Janeiro, Brasil: AIDS.

Pieper-Mooney, J. (2008). Salvar vidas y gestar la modernidad: Médicos, mujeres y Programas de Planificación Familiar en Chile. En: M. S. Zárate (Comp.) Por la salud del cuerpo. Historia y políticas sanitarias en Chile. Santiago, Chile: Universidad Alberto Hurtado.

Rubano, M. (2007). La reforma del sistema electoral chileno. Revista Estudios Constitucionales, 5(2), 365-380. DOI: $10.4067 / \mathrm{S} 0718-090 \mathrm{X} 2006000100012$

Rubin, G. (1975). The Traffic in Women: Note on the 'Political Economy' of Sex. In R. Reiter, (Ed.). Toward an Anthropology of Women. New York, USA: Monthly Review Press.

(1984). Thinking Sex: Notes for a Radical Theory of the Politics of Sexuality. In C. Vance (Ed.). Pleasure and danger: exploring female sexuality. Boston, USA: Routledge and KeganPaul.

Senado de la República de Chile (2012) Debate parlamentario en torno a la aprobación de tres proyectos de ley sobre aborto terapéutico. Legislatura $360^{\mathrm{a}}$ Sesión Ordinarias $6^{\mathrm{a}}$.

Shepard, B. (2000). The 'Double Discourse' on Sexual and Reproductive Rights in Latin America: The Chasm between Public Policy and Private Actions. Health and Human Rights, 4(2), 110-143. DOI: 10.2307/4065198

Siavelis, P.(2004). Sistema electoral, desintegración de coaliciones y democracia en Chile: ¿El fin de la Concertación? Revista de Ciencias Políticas, 24(1), 58-80. DOI: 10.4067/S0718-090X2004000100003

Valdés, T. (2013). La CEDAW y el Estado de Chile: viejas y nuevas deudas con la igualdad de género, Anuario de Derechos Humanos, 9, 171-181. DOI: 10.5354/0718-2279.2013.27042

Zárate, M.S., y González, M. (2015). Planificación familiar en la Guerra Fría chilena: política sanitaria y cooperación internacional, 1960-1973. Historia Crítica, 55, 207-230. DOI: 10.7440/histcrit55.2015.09 